\title{
Synchronous-Clock One-Way-Travel-Time Acoustic Navigation for Underwater Vehicles
}

\author{
Ryan M. Eustice \\ Department of Naval Architecture \& Marine Engineering \\ University of Michigan \\ Ann Arbor, MI 48109 \\ eustice@umich.edu
}

Hanumant Singh

Department of Applied Ocean Physics \& Engineering

Woods Hole Oceanographic Institution

Woods Hole, MA 02543

hanu@whoi.edu

\section{Louis L. Whitcomb}

Department of Mechanical Engineering

Johns Hopkins University

Baltimore, MD 21218

llw@jhu.edu

\begin{abstract}
This paper reports the development and deployment of a synchronous-clock acoustic navigation system suitable for the simultaneous navigation of multiple underwater vehicles. Our navigation system is comprised of an acoustic modem-based communication and navigation system that allows for onboard navigational data to be broadcast as a data packet by a source node, and for all passively receiving nodes to be able to decode the data packet to obtain a one-way travel time (OWTT) pseudo-range measurement and navigational ephemeris data. The reported navigation method herein uses a surface ship acting as a single moving reference beacon to a fleet of passively listening underwater vehicles. All vehicles within acoustic range are able to concurrently measure their slant range to the reference beacon using the OWTT measurement methodology, and additionally receive transmission of reference beacon position using the modem data packet. The advantages of this type of navigation system are that it can: i) concurrently navigate multiple underwater vehicles within the vicinity of the surface ship, and ii) provide a bounded-error XY position measure that is commen-
\end{abstract}


surate to conventional moored long-baseline (LBL) navigation systems (i.e., $\mathcal{O}(1 \mathrm{~m})$ ), but unlike LBL, is not geographically restricted to a fixed-beacon network. We present results for two different field experiments using a two-node configuration consisting of a global positioning system equipped surface ship acting as a global navigation aid to a Doppler-aided autonomous underwater vehicle. In each experiment, vehicle position was independently corroborated by other standard navigation means. Results for a maximum-likelihood sensor fusion framework are reported.

\section{Introduction}

Few techniques presently exist for reliable three-dimensional position sensing for underwater vehicles. Depth, altitude, heading, and roll/pitch attitude can all be instrumented with high bandwidth internal sensors. XY position, in contrast, remains difficult to instrument and is normally measured acoustically in oceanographic and commercial applications.

Conventional long-baseline (LBL) acoustic navigation systems require multiple fixed transponders - i.e., fixed or moored on the seafloor (Whitcomb et al., 1998; Hunt et al., 1974), on the hull of a surface ship (Milne, 1983), or on sea-ice (Bellingham et al., 1994). With a maximum acoustic range of 5-10 km, fixed LBL networks can cover geographically limited mission areas. Moreover, the majority of commercially available LBL navigation systems available over the last 30 years were designed to use pulsed narrowband continuous waveforms (Hunt et al., 1974). This design inherently limits an LBL net to the navigation of one vehicle per interrogation/response acoustic cycle, which is usually scheduled through a time division multiple access (TDMA) schedule to avoid acoustic interference. While this limitation is acceptable for single vehicle deployments, it is less desirable for multi-vehicle deployments because the interrogation-response navigation update period increases linearly with the number of vehicles (thereby proportionally decreasing each vehicle's overall navigation update rate). In practice, this limits tone-burst LBL navigation to networks of a few vehicles.

The recent commercial advent of wideband LBL navigation systems (Poole, 2007) relaxes the multi-vehicle navigation limitations exhibited by narrowband LBL systems. Wideband LBL uses coded broadband signals enabling it to support around 10 simultaneous acoustic channels for multi-vehicle use. However, like narrowband LBL systems, there still remains significant effort involved in deploying, calibrating, and recovering the moored fixed transponder beacons. LBL's existing prevalence within the oceanographic community is 
due to a lacuna of alternative means for obtaining bounded-error sub-sea XY position.

While the advent of the global positioning system (GPS) provides bounded-error terrestrial navigation for both surface and air vehicles, seawater is opaque to the radio-frequencies upon which GPS relies and, thus, GPS cannot be used by submerged underwater vehicles. Though ultra-short-baseline (USBL) acoustic navigation systems are preferred for short-range navigation, they are of limited usefulness for long-range navigation (Singh et al., 1996; Smith and Kronen, 1997) and, furthermore, also suffer from a TDMA update problem similar to that of LBL. Hence, neither GPS nor USBL solve the underwater vehicle XY navigation issue as outlined.

The high cost and power consumption of inertial navigation systems (INSs) has, until now, precluded their widespread use in non-military undersea vehicles. Compact $(<10$ inch diameter), low-cost $(<\$ 150 \mathrm{k})$, lowpower $(<15 \mathrm{~W})$ INSs have become commercially available within the last decade (Larsen, 2000a; Gaiffe, 2002), offering an alternative method for instrumenting absolute XYZ displacement. Modern INS position error is on the order of $1 \%$ of path-length and, when properly calibrated and aided by a Doppler velocity $\log (\mathrm{DVL})$, can approach better than $0.1 \%$ of path-length. However, as with any open-loop navigation system, error accumulation is monotonic and hence INS alone is inadequate to support the needs of longrange bounded-error navigation. For example, the path length of a vehicle traveling 3 knots for 48 hours is $144 \mathrm{~nm}(266 \mathrm{~km})$, resulting in an INS position error of $1.4 \mathrm{~nm}(2.6 \mathrm{~km})$. This may be unacceptably large depending upon the mission requirements. For a survey of current underwater navigation technologies, the reader is referred to (Kinsey et al., 2006).

In this paper we pursue the development of a synchronous-clock acoustic modem-based navigation system capable of supporting multi-vehicle bounded-error navigation over linear length scales not practically covered by traditional fixed-beacon LBL navigation means. Our navigation framework employs WHOI MicroModems (Freitag et al., 2005b,a), an underwater acoustic modem developed by Woods Hole Oceanographic Institution (WHOI), in conjunction with commercial off-the-shelf (COTS) low-power stable clocks to yield a navigation system capable of inter-vehicle communication and one-way travel time (OWTT) ranging.

Previous work using modems and synchronous-clock navigation has been reported in (Curcio et al., 2005; Bahr and Leonard, 2006) for autonomous surface-kayaks. In their work, each kayak is equipped with a tethered WHOI Micro-Modem and GPS receiver, which they use to provide a common timebase for intervehicle synchronous ranging. Since the kayaks are always on the water surface, their work did not have to address the challenges associated with implementing a synchronous-clock navigation framework on submerged 
vehicle nodes (where the use of GPS is precluded). Using an early predecessor of the modern WHOI Micro-Modem, synchronous-pinger OWTT navigation was reported in (Singh et al., 2001) where integrated range-rate positioning was used for autonomous underwater vehicle (AUV) docking. In that work, the absolute initial estimate of AUV position was obtained using an LBL fix, and was then subsequently updated using integrated range-rate measurements from the synchronous pinger to provide a dock-relative navigation correction.

Non-modem synchronous-clock OWTT ranging has been reported in (Hunt et al., 1974) for "in-hull navigation" of the manned deep-submergence vehicle Alvin. The navigation system consisted of two moored, precisely timed, pinging reference beacons synchronized to a master clock on Alvin. Reception of the transmitted beacon pulses, as measured with respect to Alvin's master clock, allowed the submersible to measure the OWTT to each beacon and hence triangulate to get a position fix. Other non-modem synchronous-clock navigation technology is that of the RAFOS oceanographic floats (Rossby et al., 1986), which employ precisely timed acoustic transmissions over very long acoustic paths to provide a position update once every two hours (or so) with a navigation error approaching one second $(1500 \mathrm{~m})$ over periods of several months. Other related work involving single transponder two-way travel-time underwater navigation has been reported in (Scherbatyuk, 1995; Larsen, 2000b; Vaganay et al., 2000; Baccou and Jouvencel, 2002; Gadre and Stilwell, 2004, 2005; Ross and Jouffroy, 2005).

In the terrestrial vehicle community there are examples of related work in range-only tracking and navigation that are tangentially applicable to our OWTT navigation paradigm. Song (1999) provides a Cramèr-Rao bound observability analysis of the range-only target tracking problem for radar and shows that if the target is moving at a constant acceleration that the observer must move with non-zero jerk to satisfy observability conditions. Similarly, Ristic et al. (2002) extend the Cramèr-Rao analysis to include the availability of not only range, but also range-rate and provide a comparison between a maximum likelihood estimate (MLE), extended Kalman filter (EKF), and regularized particle filter-concluding that the non-causal, batch MLE estimator yields best performance. Djugash and Singh (2008) devised a range-only simultaneous localization and mapping (SLAM) algorithm for radio-frequency identification (RFID) tags using a polar-representation EKF and demonstrated it to work with or without a priori knowledge of the RFID beacon locations. Also related is the work of Zhou and Roumeliotis (2008) who devised an algebraic solution to 3 degree of freedom (DOF) relative-pose estimation using vehicle-derived odometry and range-only observations between pairs of ground robots. They showed that minimally three range measurements are required to recover 3DOF robot relative-pose and that a unique solution is obtained with five or more range constraints. This 
work is most similar to our own in the type of measurement constraints that it considers (range-only and vehicle odometry); however, from their simulations Zhou and Roumeliotis showed that the algebraic solution produced large errors when corrupted with 10 centimeter-level range noise, and hence would likely perform poorly with the type of range error considered in our underwater acoustic scenario. Also important when considering the applicability of terrestrial methods to the underwater scenario is the bandwidth limited nature of the acoustic communication channel and its non-negligible propagation delay, both of which are several orders of magnitude poorer than that of electromagnetic (EM) based communication (Partan et al., 2007). EM waves are strongly attenuated underwater, and hence the assumption of reliable, instantaneous, fast communication between nodes in the network is not applicable.

In the following we describe our development of a synchronous-clock acoustic modem-based navigation system for multi-vehicle bounded-error navigation. Our contribution lies in the development of a novel timesynchronized navigation methodology, the principled formulation of how to model the one-way-travel-time constraints within a maximum likelihood estimation framework, and the experimental validation of the endto-end OWTT methodology with an AUV in a real-world setting. The rest of the paper is organized as follows. Section 2 describes our synchronous-clock implementation and methodology. Section 3 presents a sensor fusion framework for stochastically analyzing OWTT pseudo-range measurements with strap-down onboard vehicle Doppler odometry for bounded-error navigation. Section 5 reports field results for two different sets of data collected in-situ by a bottom-lock Doppler-aided AUV accompanied with OWTT pseudo-range measurements acquired from a GPS-equipped surface ship. Finally, section 6 closes with concluding remarks.

\section{Synchronous-Clock Acoustic Navigation}

Most acoustic navigation systems are based upon measuring two-way time-of-flight ranges whereby the navigation cycle consists of a vehicle-initiated interrogation pulse followed by a reply from all passively listening transponders (Hunt et al., 1974; Milne, 1983). In these systems, each vehicle in the acoustic network must interrogate the network in order to obtain a two-way time-of-flight measurement between it and all replying nodes. The advantage of such a system is that no absolute timebase is required for timeof-flight measurement; the disadvantage is that when using a narrowband acoustic navigation system, the

overall update rate for each vehicle decreases as $\frac{1}{N}$ when deploying an $N$ vehicle network. This is because narrowband designs must use a TDMA schedule to avoid acoustic interference (though, commercial wideband systems capable of supporting multiple simultaneous channels have recently become available). 


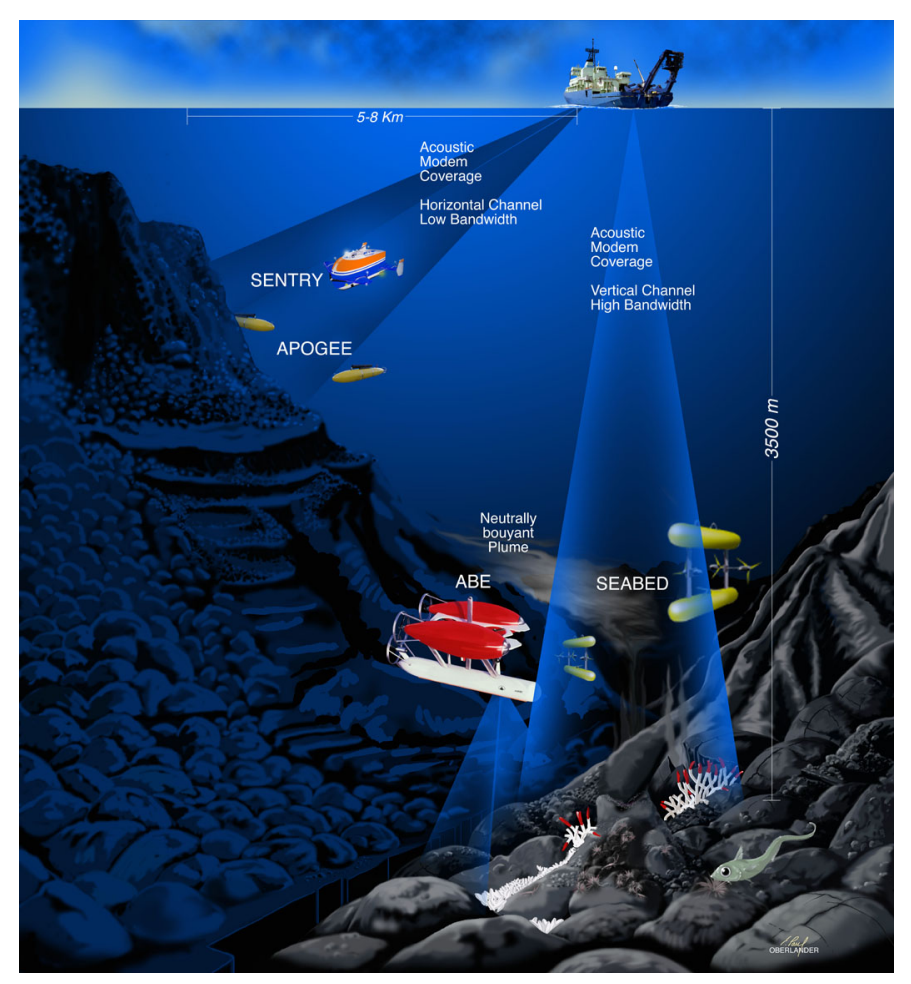

Figure 1: A depiction of the synchronous-clock one-way-travel-time navigation methodology. Here, a modemequipped surface ship acts as a roving navigation beacon to multiple, passively listening, subsea vehicles. Each transmission from the surface ship encodes time of launch and navigation data (e.g., GPS-derived ship position), which results in a slant range measurement that can be used by all receiving vehicles as a bounded-error navigation constraint.

In comparison, accurate OWTT ranging can be determined by knowing precisely the transmit and receive times of an underwater acoustic communications packet. The result is a direct one-way time-of-flight measurement from source to receiver. The advantage over two-way travel-time ranging is that OWTT ranging readily scales to a multi-vehicle environment within a master/slave architecture. This is because when a source (master) node interrogates the network, all receiving (slave) nodes can passively measure the one-way time-of-flight between each of them and the source node. Hence, in an $N$ vehicle environment, the overall update rate for each vehicle remains constant. The disadvantage, however, is increased complexity in hardware design since all nodes must carry their own synchronized stable clock hardware.

\subsection{Methodology}

Our methodology is to use OWTT ranging capabilities in the context of a surface-ship acting as a moving beacon (Figure 1). For this purpose, we employ the synchronous-transmission capabilities of the WHOI Micro-Modem (Singh et al., 2006; Freitag et al., 2005a,b). The synchronous-transmission feature of the 


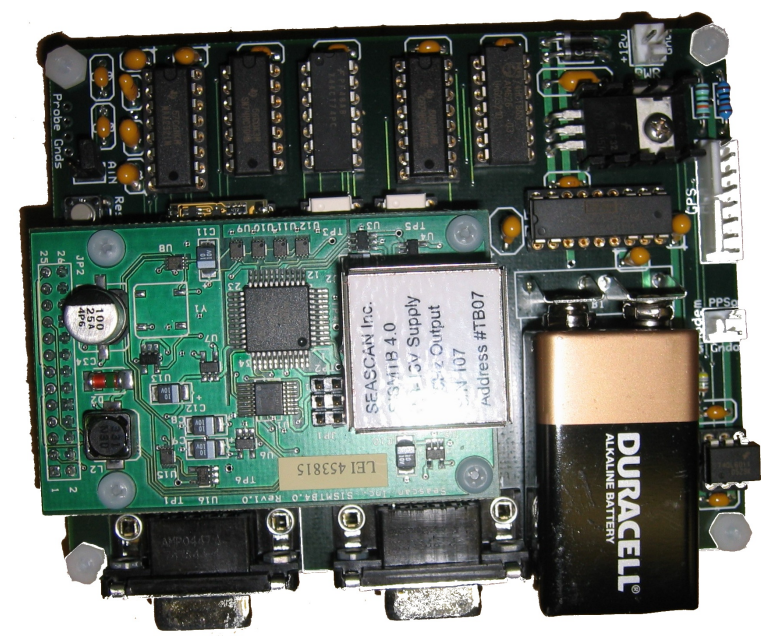

(a) 1st generation.

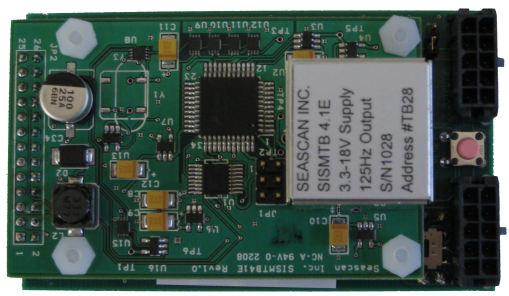

(b) 2nd generation.

Figure 2: A depiction of a first and second generation PPSBOARD designed by the authors. The PPSBOARD integrates a COTS temperature compensated crystal oscillator with a micro-controller based printed circuit board. The PPSBOARD allows for higher-level clock functionality such as PPS synchronization control, measuring of pre and post dive clock drift, and generating output suitable for real-time clock stabilization on the host PC via the open-source network time protocol (NTP) algorithm.

WHOI Micro-Modem allows it to directly and accurately measure time-of-arrival (TOA) to within $125 \mu \mathrm{s}$ (i.e., $18.75 \mathrm{~cm}$ range discretization at $1500 \mathrm{~m} / \mathrm{s}$ sound speed) between a source and receiver using a user supplied external pulse per second (PPS) reference clock. This common synchronous timebase allows for a synchronous modem communication/navigation system whereby navigation data packets can encode time of origin information as well as local navigation data such as the sender's XY and depth positional data and associated error metric. Navigation packets can be broadcast to the vehicle network, allowing all receiving nodes to passively measure the elapsed time-of-flight between source node and destination. The OWTT pseudo-range knowledge, when used in conjunction with the decoded navigation data and other onboard vehicle navigation data, provides a mechanism for bounded-error self-localization.

In our method, a ship maneuvers with an AUV fleet, tending to vehicle launch/recovery support, while also acting as a global navigation aid by broadcasting GPS-derived ship-transducer position to the vehicle network. All vehicles that are within acoustic range of the ship and which passively receive the ship navigational data can then use this knowledge to compute a running position fix and correct accumulated dead-reckoning error. For this purpose, we anticipate that vehicles will be instrumented with a standard suite of oceanographic navigation sensors including pressure depth, attitude, DVL, and possibly an INS. 
Table 1: Commercially available oscillators suitable for AUV applications.

\begin{tabular}{|l|c|l|l|l|l|}
\hline Manufacturer & Model & Type & Drift & Power & Size \\
\hline SeaScan, Inc. & SISMTB ver 4.0 & TXCO & $20 \mathrm{~ns} / \mathrm{s}$ & $5 \mathrm{~mW}$ & $71 \times 45 \times 23 \mathrm{~mm}$ \\
\hline A.C.S.A. & GIB-USC-2-A & TXCO & $20 \mathrm{~ns} / \mathrm{s}$ & $600 \mathrm{~mW}$ & $148 \times 94 \times 34 \mathrm{~mm}$ \\
\hline Trimble Navigation Ltd. & Mini-T & OXCO & $2.8 \mathrm{~ns} / \mathrm{s}$ & $1.75 \mathrm{~W}$ & $128 \times 27 \times 19 \mathrm{~mm}$ \\
\hline
\end{tabular}

\subsection{Implementation}

In our system, each submerged node (i.e., vehicle) is equipped with a COTS low-power temperature compensated crystal oscillator (TXCO) manufactured by SeaScan Inc. Other recent commercially available oscillators, such as those listed in Table 1, could also be considered for use in the OWTT methodology. For our AUV application, the SeaScan TXCO was chosen for its extreme low power $(5 \mathrm{~mW})$ combined with its sufficiently accurate free-running capability - the SeaScan TXCO is capable of providing a $20 \mathrm{~ns} / \mathrm{s}$ (typical) (i.e., a drift-rate of about $1 \mathrm{~ms}$ per $14 \mathrm{~h}$ ) reference pulse at the rate of 1 PPS over the calibrated temperature range of -5 to $35{ }^{\circ} \mathrm{C}$. This translates into a maximum per-day drift-induced range-error of $2.6 \mathrm{~m}$, which is commensurate with standard $12 \mathrm{kHz}$ LBL. The TXCO is naturally free-running, therefore, we designed a micro-controller based daughter card, called a PPSBOARD, to provide higher-level clock functionality (Figure 2).

The PPSBOARD mates to the TXCO and controls synchronization of the TXCO's PPS to an absolute reference clock as well as measures the TXCO's PPS offset with respect to the reference clock. This capability allows us to synchronize the TXCO to a common timebase at the beginning of a mission while on the surface, such as GPS-derived Coordinate Universal Time (UTC), and then measure the pre and post dive drift of the TXCO, which is useful for measuring clock drift. Additionally, the PPSBOARD generates a network time protocol (NTP) (Mills, 2006) compliant string and waveform that can be used to keep real-time clock drift onboard the vehicle host PC to within sub-milliseconds of absolute time. This level of accuracy is sufficient for the host PC to be able to assign which second corresponds to which TXCO PPS pulse when communicating with the Micro-Modem. This time reference is then encoded as the time of origin in the acoustic navigation packet.

The surface ship uses a COTS GPS-based network timeserver for a stable clock source. The unit, manufactured by Meinberg Inc., uses a high-quality oven compensated crystal oscillator (OXCO) with a GPSsynchronous accuracy of $0.005 \mathrm{~ns} / \mathrm{s}$ and a free-running accuracy of $1.5 \mathrm{~ns} / \mathrm{s}$. Hence, the ship-based timeserver can be considered essentially drift-free and only the vehicle TXCO drift must be accounted for when computing pseudo-range measurements from time-of-flight data. 


\subsection{TXCO PPS Drift Characterization}

To characterize the drift performance of the TXCO used on sub-sea vehicle nodes, we performed an experiment consisting of 40 trials whereby we synchronized and recorded the free-run drift of the PPSBOARD over a 24-hour period in an ambient room temperature environment. For comparison, we used the Meinberg's PPS signal as ground-truth. Figure 3 summarizes the outcome of the 40 trials and displays measured drift, sample statistics, and recorded ambient temperature for each run. In particular, note in Figure 3(a) the time-varying drift phenomenon of the TXCO PPS. Qualitatively, the time-varying nature of the sample paths may, at first glance, appear similar to that of a random-walk process; however, note that each trial steadily increases in drift (positively or negatively) without significant random fluctuation. Hence, it does not exhibit Brownian motion. Furthermore, Figure 3(c) quantitatively displays the associated sample variance and standard deviation across the trials where we see that the sample variance does not exhibit a linear growth with time as would be the case if it were indeed a random-walk process. Instead it appears to exhibit quadratic growth with time (linear standard deviation). At this point in time, further analysis is required to properly model clock drift.

The maximum drift error across all trials in Figure 3(a) is under $300 \mu$ s, which translates into a driftinduced range bias of less than $0.45 \mathrm{~m}$ over a $24 \mathrm{~h}$ period. While the reported experiments here were for an ambient temperature environment, we have also conducted some preliminary studies in which we immersed the TXCO into a controlled temperature-bath-exposing it to a step-response temperature change. The experimental setup consisted of synchronizing the PPSBOARD to the Meinberg at ambient room temperature (approximately $24{ }^{\circ} \mathrm{C}$ ) and then inserting the PPSBOARD into a temperature-controlled refrigerator set at approximately $4{ }^{\circ} \mathrm{C}$ and allowing it to stabilize. We noted the PPSBOARD's drift relative to the Meingberg reference PPS and observed that the magnitude of error drift remained within the manufacturer's reported error tolerance of $20 \mathrm{~ns} / \mathrm{s}$. The manufacturer's technical specification for the TXCO is $20 \mathrm{~ns} / \mathrm{s}$ (typical) over the calibrated temperature range of -5 to $35{ }^{\circ} \mathrm{C}$. This is relevant because PPS synchronization will typically occur at the surface prior to launch, meaning that the vehicle electronics and enclosed TXCO will go through a temperature gradient between ambient air at the surface to seafloor water temperature at depth. So while we should expect the drift performance of the TXCO to degrade with respect to the controlled laboratory results reported here, it still suggests that for relatively short-duration missions (i.e., several hours in length) drift-induced range-bias will be a negligent error source in overall underwater vehicle navigation. 


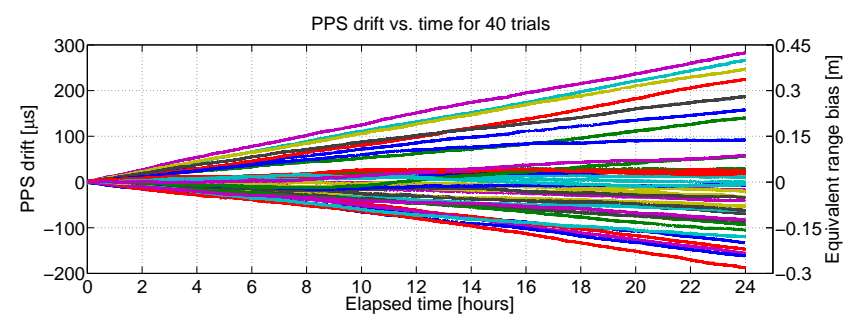

(a)

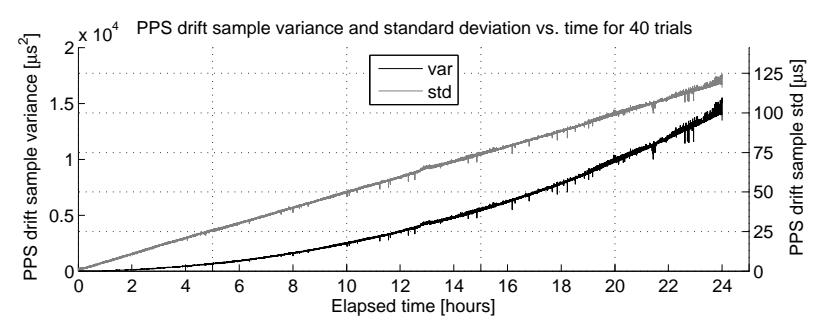

(c)

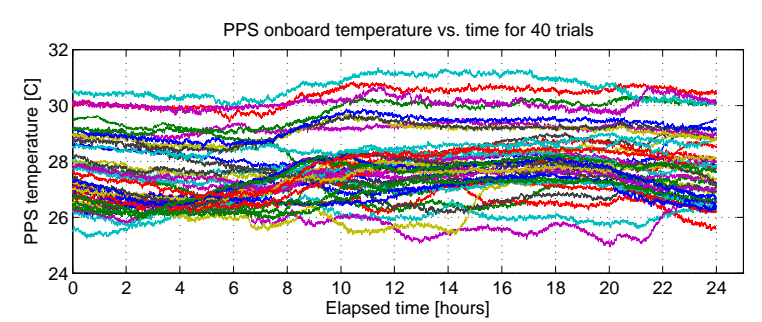

(b)

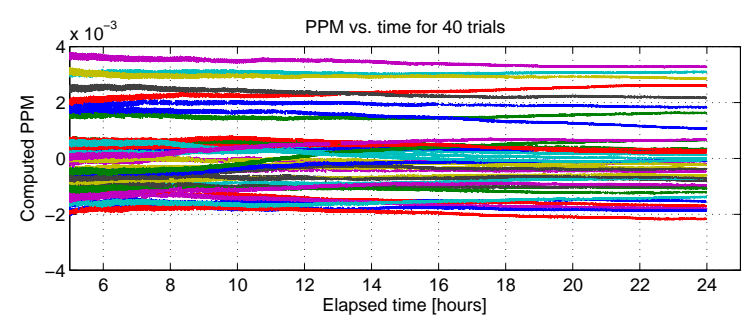

(d)

Figure 3: PPS drift characterization; benchmark results for 40 individual trials. Each trial was conducted over a 24-hour period at ambient room temperature. The time axis shows the elapsed time in hours since synchronization of the PPSBOARD with respect to a reference drift-free PPS. (a) Measured PPS drift for all trials; each sample path is color coded by trial number. The y-axis on the left reports the measured offset in microseconds while the axis on the right reports it in range equivalent meters (assuming a sound speed of $1500 \mathrm{~m} / \mathrm{s}$ ). (b) Measured onboard TXCO temperature; fluctuation is about normal ambient room temperature. (c) PPS drift sample variance and standard deviation across all trials. (d) Measured PPS drift for all trials, reported in units of parts-per-million (PPM). 


\section{Maximum Likelihood Fusion Framework}

In this section, we describe a maximum-likelihood sensor fusion framework for bounded-error XY vehicle navigation that combines: 1) vehicle-derived inter-ping Doppler odometry, 2) ship GPS-derived position, and 3) OWTT pseudo-range measurements between ship and vehicle. The framework is suitable for offline batch post-processing for the purposes of optimal re-navigation, and can also be extended to in-situ vehicle use by selecting an appropriate sliding time window of most recent data.

\subsection{Assumptions}

In the forthcoming formulation we make the following assumptions:

1. First, that dives are relatively short in length (i.e., on the order of several hours) such that PPS driftinduced range-bias remains negligible over the course of the dive and, therefore, can be neglected.

2. Second, that the sound speed profile is locally homogeneous within the prescribed bounding box of vehicle operations. This implies that time-of-flight measurements can be converted to pseudoranges via a linear scaling. In practice this is satisfied by conducting a pre-dive local conductivitytemperature-depth (CTD) cast and using a depth-weighted averaged sound velocity for scaling.

3. Third, that the estimation problem can be reduced to that of XY horizontal plane dynamics only. For this purpose, we assume that the vehicle is equipped with a pressure depth sensor of sufficient accuracy such that slant-range pseudo-ranges can be projected onto the horizontal plane. This also requires that the ship's trajectory never pass directly over top the vehicle, which would otherwise introduce a singularity into the horizontal range projection.

4. Fourth, that the vehicle is capable of measuring its own dead-reckoned (DR) XY odometry and error covariance in-between OWTT pings. For example, this could be obtained from bottom-lock Doppler velocity data, an onboard INS system, or a vehicle dynamic model.

5. Finally, in the current formulation, we assume that all OWTT pseudo-range measurements occur between surface ship and vehicle only. At present, we do not consider inter-vehicle OWTT ranging. Hence, it is sufficient that each vehicle only track ship trajectory for the purposes of self-localization. 


\subsection{State Description}

We denote topside ship trajectory as $\mathbf{x}_{s}(t)=\left[x_{s}(t), y_{s}(t)\right]^{\top}$, where $\left(x_{s}(t), y_{s}(t)\right)$ represents XY ship transducer position in a locally-defined, Earth-tangent, Cartesian coordinate frame. Similarly, we denote bottomside vehicle trajectory as $\mathbf{x}_{v}(t)=\left[x_{v}(t), y_{v}(t)\right]^{\top}$. In the aforementioned scenario, the ship initiates a OWTT broadcast at time $t_{s}$ while the vehicle receives that same broadcast at a corresponding latter time $t_{v}>t_{s}$. For a given OWTT range measurement, $z_{r_{i}}$, indexed by subscript $i$, we note that this measurement actually corresponds to a ship/vehicle sample pair, $\mathbf{x}_{s_{i}}$ and $\mathbf{x}_{v_{i}}$, each sampled at distinct times $\mathbf{x}_{s_{i}}=\mathbf{x}_{s}\left(t_{s_{i}}\right)$ and $\mathbf{x}_{v_{i}}=\mathbf{x}_{v}\left(t_{v_{i}}\right)$, respectively. For notational convenience, we drop the explicit dependence on time and instead implicitly embed sample time within the $\mathbf{x}_{s_{i}}$ and $\mathbf{x}_{v_{i}}$ sample index notation. Figure 4 depicts a sequence of OWTT pseudo-range measurements occurring between ship and vehicle.

Next, we write our measurement observation models in terms of this sample index state description.

\subsubsection{OWTT Pseudo-Range Observation Model}

As previously stated, we use measured vehicle depth to horizontally project our raw time-of-flight pseudorange measurements to the $\mathrm{XY}$ horizontal plane. Using this simplification, we write the horizontal range measurement as:

$$
z_{r_{i}}=\left\|\mathbf{x}_{v_{i}}-\mathbf{x}_{s_{i}}\right\|+w_{r_{i}}
$$

where $w_{r_{i}}$ is an additive noise term that accounts for measurement error. For the current exposition we model $w_{r_{i}}$ as being normally distributed with $w_{r_{i}} \sim \mathcal{N}\left(0, \Sigma_{r_{i}}\right)$ with $\mathrm{E}\left[w_{r_{i}} w_{r_{j}}\right]=0$ for all $i \neq j$.

\subsubsection{GPS-derived Ship Position Observation Model}

We first transform raw GPS measured latitude and longitude to the locally referenced XY coordinate system and then linearly interpolate to sample time $t_{s_{i}}$. Based upon this we write our ship GPS observation model as:

$$
\mathbf{z}_{g_{i}}=\mathbf{x}_{s_{i}}+\mathbf{w}_{g_{i}}
$$

where $\mathbf{w}_{g_{i}}$ is an additive noise term that accounts for measurement error. We model this as being normally distributed with $\mathbf{w}_{g_{i}} \sim \mathcal{N}\left(0, \Sigma_{g_{i}}\right)$ and $\mathrm{E}\left[\mathbf{w}_{g_{i}} \mathbf{w}_{g_{j}}^{\top}\right]=0$ for all $i \neq j$. 


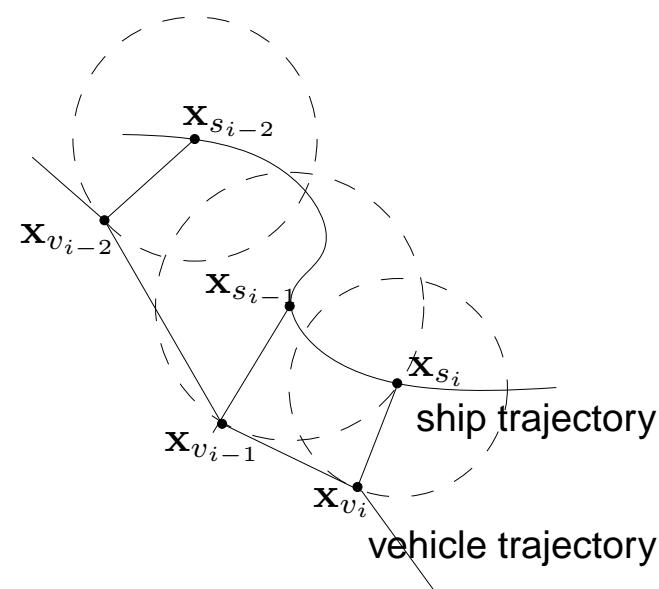

(a)

\begin{tabular}{|l|l|}
\hline Measurement Origin & Observation Model \\
\hline \hline OWTT Pseudo-Range & $\mathbf{z}_{r_{i}}=\left\|\mathbf{x}_{v_{i}}-\mathbf{x}_{s_{i}}\right\|+w_{r_{i}}$ \\
Ship GPS Position & $\mathbf{z}_{g_{i}}=\mathbf{x}_{s_{i}}+\mathbf{w}_{g_{i}}$ \\
Vehicle Odometry & $\mathbf{z}_{o_{i}}=\left(\mathbf{x}_{v_{i}}-\mathbf{x}_{v_{i-1}}\right)+\mathbf{w}_{o_{i}}$ \\
\hline
\end{tabular}

(b)

Figure 4: Maximum likelihood estimate formulation. (a) A depiction of the 2D OWTT range geometry where $\mathbf{x}_{s_{i}}$ and $\mathbf{x}_{v_{i}}$ denote corresponding samples from the ship and vehicle trajectories, respectively. (b) A table of the available measurements and their relation to state entries. The $w_{r_{i}}, \mathbf{w}_{g_{i}}$, and $\mathbf{w}_{o_{i}}$ terms each represent additive measurement noise.

\subsubsection{Vehicle-derived Odometry Observation Model}

Vehicle-derived odometry measurements represent the inter-ping vector displacement between OWTT fixes and are a necessary constraint in order to establish vehicle observability when dealing with single-transponder ranges (Ross and Jouffroy, 2005; Gadre and Stilwell, 2004). We write our odometry observation model as:

$$
\mathbf{z}_{o_{i}}=\mathbf{x}_{v_{i}}-\mathbf{x}_{v_{i-1}}+\mathbf{w}_{o_{i}}
$$

where $\mathbf{w}_{o_{i}}$ is an additive noise term that accounts for measurement error. We model the error as being normally distributed with $\mathbf{w}_{o_{i}} \sim \mathcal{N}\left(0, \Sigma_{o_{i}}\right)$ and $\mathrm{E}\left[\mathbf{w}_{o_{i}} \mathbf{w}_{o_{j}}^{\top}\right]=0$ for all $i \neq j$.

\subsection{Maximum Likelihood Estimator}

We pose sensor fusion as a MLE optimization problem. In this context, we treat ship/vehicle sample pairs as unknown parameters that we wish to estimate. To begin, we consider the case of $n$ range measurements 
and define the following:

$$
\begin{array}{ll}
\mathbf{X}_{v}=\left\{\mathbf{x}_{v_{i}}\right\}_{0}^{n-1} & \text { is the set of vehicle trajectory samples, } \\
\mathbf{X}_{s}=\left\{\mathbf{x}_{s_{i}}\right\}_{0}^{n-1} & \text { is the set of ship trajectory samples, } \\
\mathbf{Z}_{r}=\left\{z_{r_{i}}\right\}_{0}^{n-1} & \text { is the set of pseudo-range measurements, } \\
\mathbf{Z}_{g}=\left\{\mathbf{z}_{g_{i}}\right\}_{0}^{n-1} & \text { is the set of ship GPS measurements, } \\
\mathbf{Z}_{o}=\left\{\mathbf{z}_{o_{i}}\right\}_{1}^{n-1} & \text { is the set of odometry measurements }
\end{array}
$$

with $\mathbf{X}=\left\{\mathbf{X}_{v}, \mathbf{X}_{s}\right\}$ and $\mathbf{Z}=\left\{\mathbf{Z}_{r}, \mathbf{Z}_{g}, \mathbf{Z}_{o}\right\}$

Denoting the measurement likelihood as $L(\mathbf{X})$ we have

$$
\begin{aligned}
L(\mathbf{X}) & =p(\mathbf{Z} \mid \mathbf{X}) \\
& =p\left(\mathbf{Z}_{r} \mid \mathbf{X}_{v}, \mathbf{X}_{s}\right) p\left(\mathbf{Z}_{g} \mid \mathbf{X}_{s}\right) p\left(\mathbf{Z}_{o} \mid \mathbf{X}_{v}\right),
\end{aligned}
$$

where the mutual independence of measurements on parameters is explicit. To optimize, we wish to find

$$
\hat{\mathbf{X}}=\arg \max _{\mathbf{X}} p(\mathbf{Z} \mid \mathbf{X})
$$

which is equivalent to solving

$$
\hat{\mathbf{X}}=\arg \min _{\mathbf{X}}-\ln p(\mathbf{Z} \mid \mathbf{X})
$$

Under the assumed observation models and noise statistics, we can write our objective function, $C(\mathbf{X})$, as

$$
\begin{aligned}
C(\mathbf{X}) & =-\ln p(\mathbf{Z} \mid \mathbf{X}) \\
& =\frac{1}{2} \sum_{i=0}^{n-1}\left(z_{r_{i}}-\left\|\mathbf{x}_{v_{i}}-\mathbf{x}_{s_{i}}\right\|\right)^{\top} \Sigma_{r_{i}}^{-1}\left(z_{r_{i}}-\left\|\mathbf{x}_{v_{i}}-\mathbf{x}_{s_{i}}\right\|\right)+\frac{1}{2} \sum_{i=0}^{n-1}\left(\mathbf{z}_{g_{i}}-\mathbf{x}_{s_{i}}\right)^{\top} \Sigma_{g_{i}}^{-1}\left(\mathbf{z}_{g_{i}}-\mathbf{x}_{s_{i}}\right) \\
& +\frac{1}{2} \sum_{i=1}^{n-1}\left(\mathbf{z}_{o_{i}}-\left(\mathbf{x}_{v_{i}}-\mathbf{x}_{v_{i-1}}\right)\right)^{\top} \Sigma_{o_{i}}^{-1}\left(\mathbf{z}_{o_{i}}-\left(\mathbf{x}_{v_{i}}-\mathbf{x}_{v_{i-1}}\right)\right) .
\end{aligned}
$$

Furthermore, this can be written compactly as

$$
C(\mathbf{X})=\left(\mathbf{Z}-\mathbf{h}_{\mathbf{Z}}(\mathbf{X})\right)^{\top} \Sigma_{\mathbf{Z}}^{-1}\left(\mathbf{Z}-\mathbf{h}_{\mathbf{Z}}(\mathbf{X})\right)
$$

where $\mathbf{h}_{\mathbf{Z}}(\mathbf{X})$ is the stacked vector of observations and $\Sigma_{\mathbf{Z}}$ is the block-diagonal measurement covariance. 
The above formulation amounts to a nonlinear weighted least-squares optimization problem. To solve, we employ the Levenberg-Marquardt algorithm (Press et al., 1992) starting with an initial guess of DR vehicle position and GPS-measured ship position. A first-order estimate of parameter covariance, $\Sigma_{\mathbf{X}}$, can then be obtained by evaluating the Jacobian at the optima,

$$
\mathrm{J}=\left.\frac{\mathrm{d} \mathbf{h}_{\mathbf{Z}}(\mathbf{X})}{\mathrm{d} \mathbf{X}}\right|_{\hat{\mathbf{X}}}
$$

and computing (Haralick, 1998):

$$
\Sigma_{\mathbf{X}}=\left(\mathrm{J}^{\top} \Sigma_{\mathbf{Z}}^{-1} \mathrm{~J}\right)^{-1}
$$

\section{DVL-derived Odometry Covariance}

The MLE formulation assumes that measurement covariances are available. For GPS measurements, most receivers are capable of reporting a quality of fix, which can be used to derive a covariance estimate; for OWTT ranges, we can estimate TOA precision by measuring the sample jitter between two fixed nodes. The only remaining item is to assign an appropriate covariance measure to our inter-ping odometry samples. In the following, we derive a theoretical first-order calculation of covariance for bottom-lock DVL XY displacement.

\subsection{Theoretical First-Order Covariance Estimate}

Integrated Doppler XY displacement between sample times $t_{v_{i-1}}$ and $t_{v_{i}}$, under the assumption of negligible pitch and roll, can be written as

$$
\mathbf{x}_{o_{i-1, i}}=\int_{t_{v_{i-1}}}^{t_{v_{i}}} \mathrm{R}(\psi(t)) \mathbf{u}(t) \mathrm{dt}
$$

where $\psi(t)$ is the time-varying vehicle heading, $\mathbf{u}(t)=[u(t), v(t)]^{\top}$ is the vector velocity body-frame surge and sway, and

$$
R(\theta)=\left[\begin{array}{rr}
\cos \theta & -\sin \theta \\
\sin \theta & \cos \theta
\end{array}\right]
$$

is the orthonormal body to world rotation matrix. In practice, the DVL returns discrete-time samples, therefore, we rewrite (10) as a summation using forward Euler integration as

$$
\mathbf{x}_{o_{i-1, i}}=T \sum_{j=0}^{m-1} \mathrm{R}\left(\psi_{j}\right) \mathbf{u}_{j}
$$


where $T$ is the DVL sample period, $m=\left\lfloor\left(t_{v_{i}}-t_{v_{i-1}}\right) / T\right\rfloor$ is the number of inter-ping samples, $\mathbf{u}_{j}=\mathbf{u}\left(t_{v_{i-1}}+j T\right)$ is the sampled velocity, and $\psi_{j}=\psi\left(t_{v_{i-1}}+j T\right)$ is the sampled heading.

To proceed with a calculation of DVL displacement covariance, we model the heading and velocity as each being corrupted by zero-mean, mutually-independent, white Gaussian noise processes $w_{\psi_{j}} \sim \mathcal{N}\left(0, \sigma_{\psi_{j}}^{2}\right)$ and $\mathbf{w}_{\mathbf{u}_{j}} \sim \mathcal{N}\left(0, \Sigma_{\mathbf{u}}\right)$, respectively, which leads to a DVL odometry measurement of

$$
\mathbf{z}_{o_{i}}=T \sum_{j=0}^{m-1} \mathrm{R}\left(\psi_{j}+w_{\psi_{j}}\right)\left(\mathbf{u}_{j}+\mathbf{w}_{\mathbf{u}_{j}}\right)
$$

Assuming that the distribution on $w_{\psi_{j}}$ is sufficiently narrow, we make the small angle approximation:

$$
\begin{aligned}
\cos \left(\psi_{j}+w_{\psi_{j}}\right) & \approx \cos \psi_{j}-w_{\psi_{j}} \sin \psi_{j} \\
\sin \left(\psi_{j}+w_{\psi_{j}}\right) & \approx \sin \psi_{j}+w_{\psi_{j}} \cos \psi_{j}
\end{aligned}
$$

and apply it within the DVL odometry model as

$$
\begin{aligned}
\mathbf{z}_{o_{i}} & \approx T \sum_{j=0}^{m-1}\left[\mathrm{R}\left(\psi_{j}\right)-w_{\psi_{j}} \mathrm{R}^{\prime}\left(\psi_{j}\right)\right]\left(\mathbf{u}_{j}+\mathbf{w}_{\mathbf{u}_{j}}\right) \\
& =\underbrace{T \sum_{j=0}^{m-1} \mathrm{R}\left(\psi_{j}\right) \mathbf{u}_{j}}_{\mathbf{x}_{o_{i-1, i}}}+\underbrace{T \sum_{j=0}^{m-1} \mathrm{R}\left(\psi_{j}\right) \mathbf{w}_{\mathbf{u}_{j}}}_{\mathrm{S}_{1}}-\underbrace{T \sum_{j=0}^{m-1} w_{\psi_{j}} \mathrm{R}^{\prime}\left(\psi_{j}\right) \mathbf{u}_{j}}_{\mathrm{S}_{2}}-T \underbrace{\sum_{j=0}^{m-1} w_{\psi_{j}} \mathrm{R}^{\prime}\left(\psi_{j}\right) \mathbf{w}_{\mathbf{u}_{j}}}_{j=0} \\
& =\mathbf{x}_{o_{i-1, i}+\mathrm{S}_{1}-\mathrm{S}_{2}}^{-\mathrm{S}_{3}} \\
\text { where } \mathrm{R}^{\prime}(\theta)= & {\left[\begin{array}{rr}
-\sin \theta & -\cos \theta \\
\cos \theta & -\sin \theta
\end{array}\right] . }
\end{aligned}
$$

Taking the expectation of $(13 \mathrm{~b})$ we have $\mathrm{E}\left[\mathbf{z}_{O_{i}}\right]=\mathbf{x}_{o_{i-1, i}}$. To compute the DVL covariance we evaluate

$$
\begin{aligned}
\Sigma_{o_{i}} & =\mathrm{E}\left[\left(\mathbf{z}_{o_{i}}-\mathrm{E}\left[\mathbf{z}_{o_{i}}\right]\right)\left(\mathbf{z}_{o_{i}}-\mathrm{E}\left[\mathbf{z}_{o_{i}}\right]\right)^{\top}\right] \\
& =\mathrm{E}\left[\mathrm{S}_{1} \mathrm{~S}_{1}^{\top}\right]-\mathrm{E}\left[\mathrm{S}_{1} \mathrm{~S}_{2}^{\top}\right]-\mathrm{E}\left[\mathrm{S}_{1} \mathrm{~S}_{3}^{\top}\right]-\mathrm{E}\left[\mathrm{S}_{2} \mathrm{~S}_{1}^{\top}\right]+\mathrm{E}\left[\mathrm{S}_{2} \mathrm{~S}_{2}^{\top}\right]+\mathrm{E}\left[\mathrm{S}_{2} \mathrm{~S}_{3}^{\top}-{ }^{0}-\mathrm{E}\left[\mathrm{S}_{3} \mathrm{~S}_{1}^{\top}\right]+\mathrm{E}\left[\mathrm{S}_{3} \mathrm{~S}_{2}^{\top}\right]+\mathrm{E}\left[\mathrm{S}_{3} \mathrm{~S}_{3}^{\top}\right]\right.
\end{aligned}
$$

and note that the zero terms arise from the mutual-independence of $w_{\psi_{j}}$ and $\mathbf{w}_{\mathbf{u}_{j}}$. Evaluating the expecta- 
tions in (14) and defining $\mathrm{U}_{j}$ as

$$
\mathrm{U}_{j}=\left[\begin{array}{cc}
u_{j}^{2} & u_{j} v_{j} \\
u_{j} v_{j} & v_{j}^{2}
\end{array}\right]
$$

we arrive at the final expression for first-order DVL odometry covariance:

$$
\Sigma_{o_{i}}=T^{2} \sum_{j=0}^{m-1} \mathrm{R}\left(\psi_{j}\right) \Sigma_{\mathbf{u}_{j}} \mathrm{R}\left(\psi_{j}\right)^{\top}+T^{2} \sum_{j=0}^{m-1} \mathrm{R}^{\prime}\left(\psi_{j}\right) \mathrm{U}_{j} \mathrm{R}^{\prime}\left(\psi_{j}\right)^{\top} \sigma_{\psi_{j}}^{2}+T^{2} \sum_{j=0}^{m-1} \mathrm{R}^{\prime}\left(\psi_{j}\right) \Sigma_{\mathbf{u}_{j}} \mathrm{R}^{\prime}\left(\psi_{j}\right)^{\top} \sigma_{\psi_{j}}^{2} .
$$

\subsection{Linear Trackline Example}

To help the reader gain some intuition behind the interpretation of (15), here we consider the case of constant forward velocity under constant heading and no side-slip:

$$
\mathbf{u}_{j}=[u, 0]^{\top}, \quad \psi_{j}=\psi
$$

This configuration replicates the typical survey scenario of linear tracklines. For the purposes of demonstration, we further assume time-invariant noise statistics, and in the case of Doppler velocity, isotropic covariance:

$$
\sigma_{\psi_{j}}^{2}=\sigma_{\psi}^{2}, \quad \Sigma_{\mathbf{u}_{j}}=\sigma_{d}^{2} \mathrm{I}_{2 \times 2} .
$$

Under the above scenario, (15) reduces to

$$
\Sigma_{o_{i}}=m T^{2} \sigma_{d}^{2}\left(1+\sigma_{\psi}^{2}\right) \mathrm{I}_{2 \times 2}+m T^{2} u^{2} \sigma_{\psi}^{2}\left[\begin{array}{cc}
\sin ^{2} \psi & \sin \psi \cos \psi \\
\sin \psi \cos \psi & \cos ^{2} \psi
\end{array}\right]
$$

The first term in (16) accounts for an isotropic linear growth with time in XY DVL trajectory error. This error source is due to the random walk contribution of measured velocity error, typical of an independent increments process. The second term in (16) accounts for heading uncertainty and is dependent upon direction of travel - causing error growth transverse to the direction of motion. For example, if heading due north (i.e, $\psi=0$ ), then this term accounts for accumulated error growth in the East-West direction due to measurement uncertainty in the true heading of the vehicle. Figure 5(a) demonstrates this phenomenon for a representative set of headings where the ellipsoids have been calculated for $10 \mathrm{~m}$ of travel at $1 \mathrm{~m} / \mathrm{s}$ forward velocity with $4 \mathrm{~Hz}$ sampling at $3 \mathrm{~mm} / \mathrm{s}$ velocity precision and a heading precision of $1^{\circ}$. The ellipsoids represent 1- $\sigma$ contours and have been inflated by a factor of 1000 for visualization. 


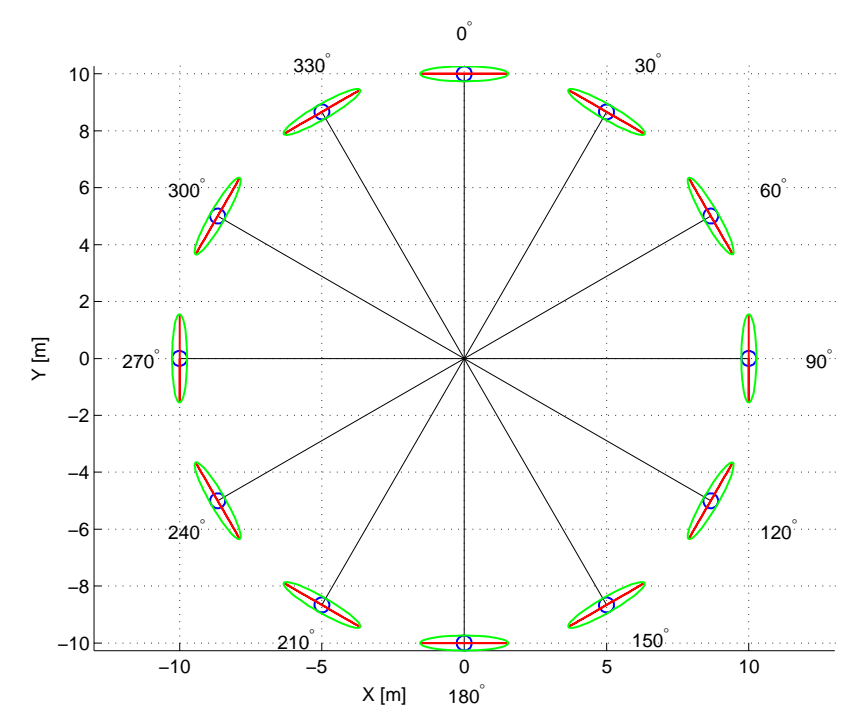

(a)
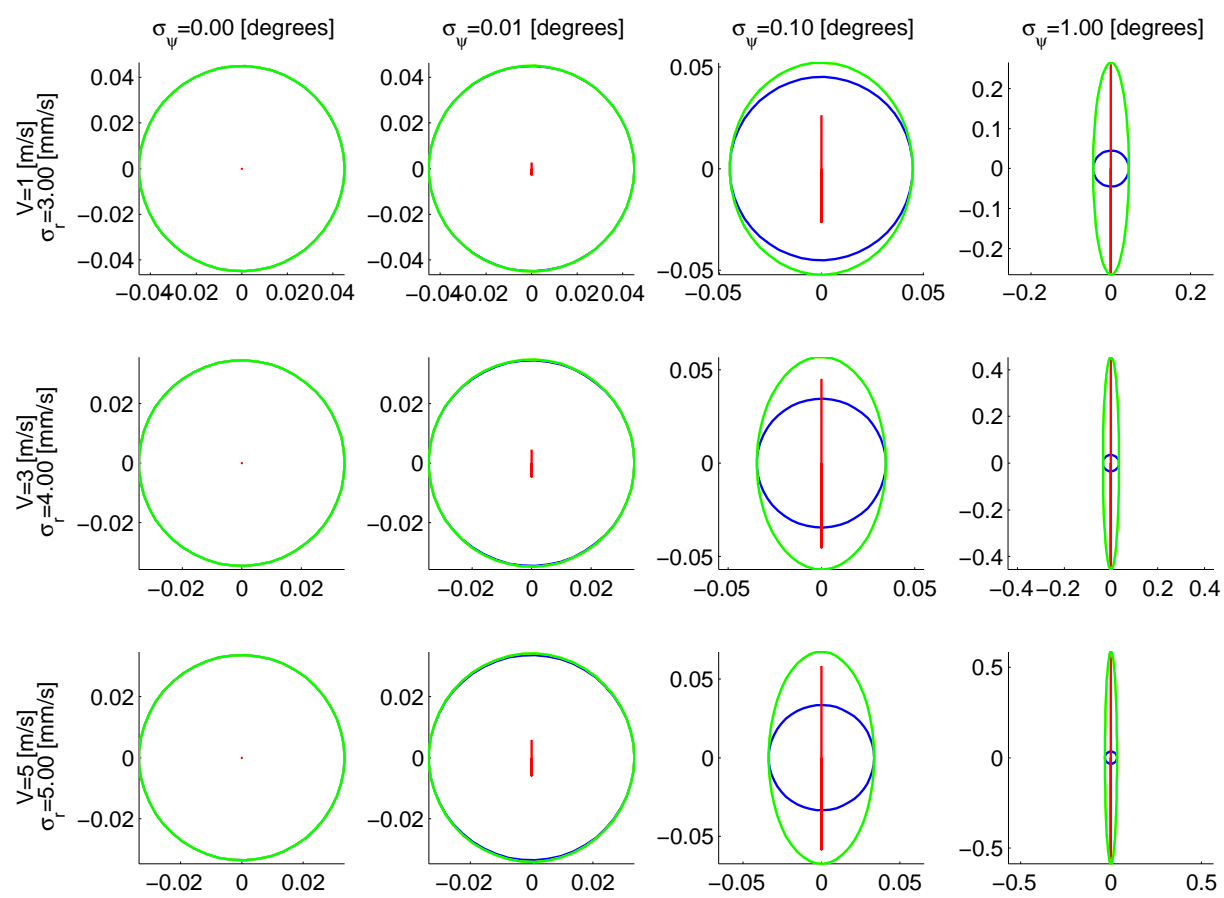

(b)

Figure 5: A demonstration of theoretical XY DVL odometry covariance for constant forward velocity with no side-slip under constant heading. (a) This figure plots the DVL covariance ellipsoid for a representative set of headings. The ellipsoids represent 1- $\sigma$ contours and have been inflated by a factor of 1000 for visualization. In blue we have the isotropic component of covariance error, in red the anisotropic component due to heading error, and in green their superposition. Note that the heading error contributes to error growth transverse to the direction of travel. (b) This figure evaluates the theoretical DVL covariance ellipsoid for a range of typical heading precisions and $1200 \mathrm{kHz}$ DVL velocity precisions for $100 \mathrm{~m}$ of path length in the East-West direction. Units shown are in meters and the ellipsoids represent 3- $\sigma$ contours. Refer to (a) for ellipse colors. 


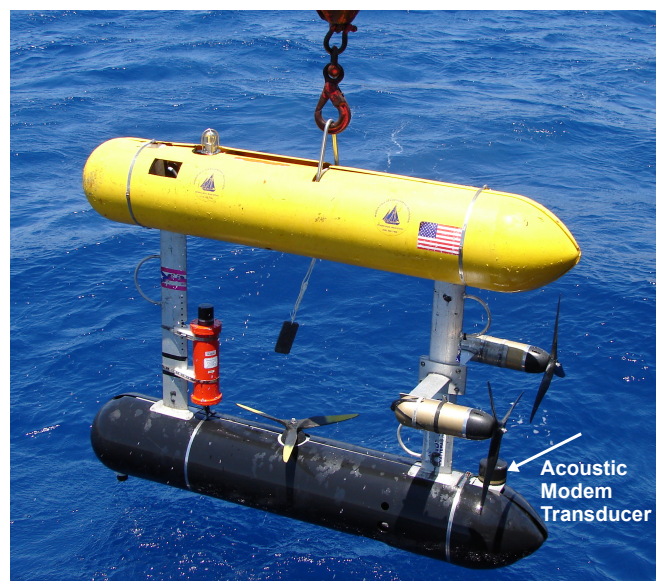

Figure 6: The SeaBED AUV as used during field experiments (Singh et al., 2004). The orange Tracklink beacon mounted on the front strut provided an independent measurement of ship-to-vehicle range.

To gain further insight into the magnitude of heading error and its effect on XY uncertainty, Figure 5(b) displays results for evaluating (16) for a sampling of typical heading and velocity errors. For this demonstration, we set $\psi=90^{\circ}$ for a path length of $100 \mathrm{~m}$ at representative speeds of $u=1,3$, and $5 \mathrm{~m} / \mathrm{s}$ and velocity standard deviations of $\sigma_{d}=3,4$, and $5 \mathrm{~mm} / \mathrm{s}$, respectively. These numbers are provided by the RDI Workhorse manual as being typical $1200 \mathrm{kHz}$ bottom-lock precisions (RD Instruments, 2001). Most noticeably, Figure 5(b) demonstrates that cross-track error is the dominating component for heading precisions above $1^{\circ}$.

\section{Results}

In this section we report results for a set of two field experiments employing OWTT navigation with MLE sensor fusion. Both experiments were for a two-node configuration consisting of the tending surface ship and a single AUV. In each case, the SeaBED AUV platform (Figure 6) was used.

\subsection{Experimental Setup}

SeaBED is instrumented with a typical suite of oceanographic navigation sensors including pressure sensor depth, $1200 \mathrm{kHz}$ DVL body-frame velocities, an IXSEA North-seeking 3-axis fiber optic gyrocompass for attitude, and a PPS-capable WHOI Micro-Modem (Singh et al., 2004). In addition, we integrated our PPSBOARD and a Garmin GPS-16HVS GPS receiver into the vehicle system so that we could conduct OWTT experiments. The Garmin GPS unit outputs a 1 PPS reference signal accurate to within $1 \mu$ s of 


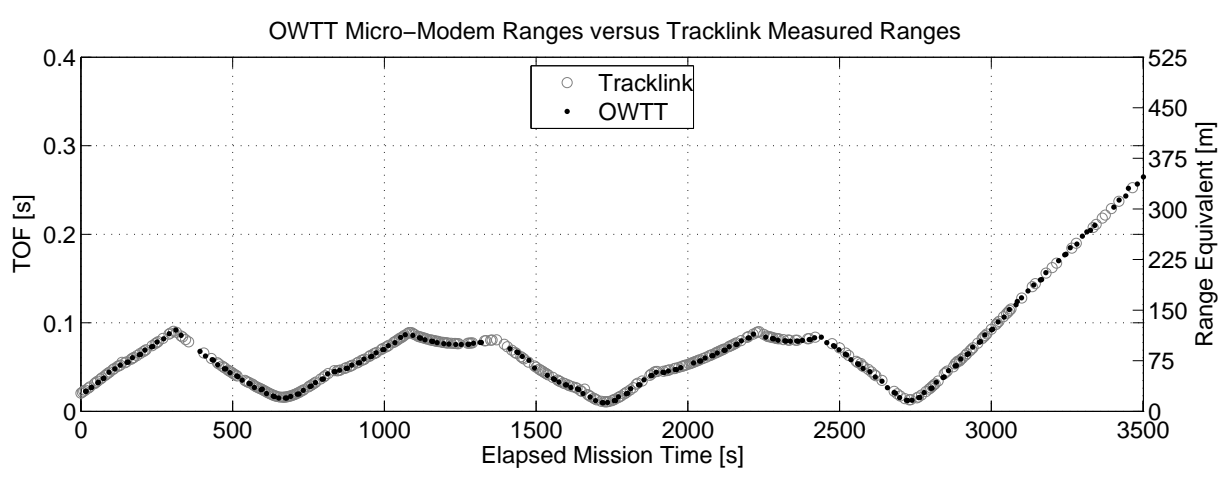

(a)

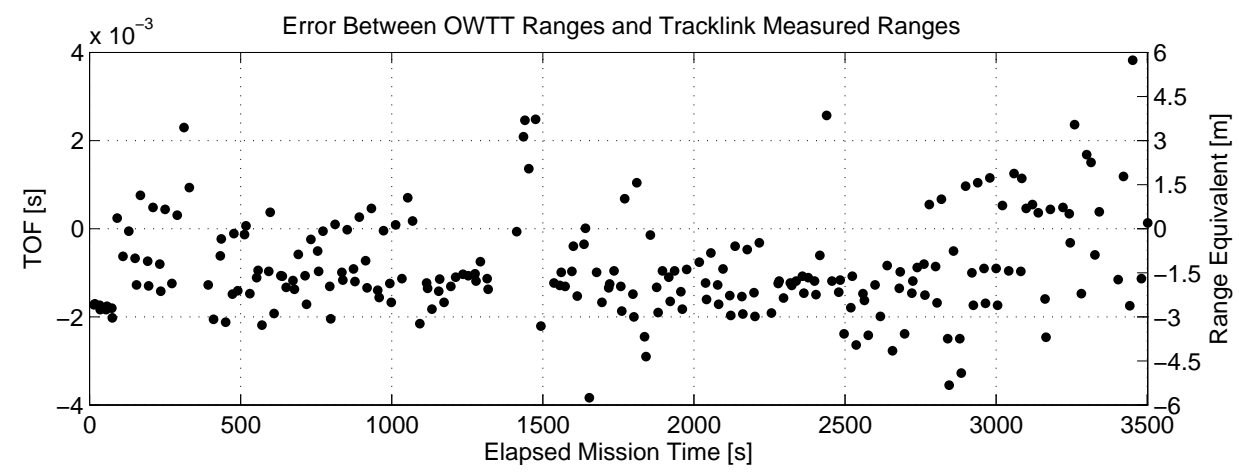

(b)

Figure 7: A comparison of modem-derived OWTT ranges versus USBL two-way travel-time ranges measured between ship and AUV. (a) A plot of raw ranges as measured by the the Tracklink USBL system and modemderived OWTTs. (b) A comparison of the time-of-flight discrepancy obtained by linearly interpolating the USBL range data to the OWTT timebase. For reference, the y-axis scale on the right shows the equivalent range error assuming a sound speed of $1500 \mathrm{~m} / \mathrm{s}$.

UTC when it has GPS lock. We use this PPS reference for pre-dive time synchronization of the free running TXCO onboard the AUV while at the surface.

The surface ship is equipped with a PPS-capable Micro-Modem, a GPS receiver used for measuring ship transducer position, and a Meinberg GPS-based NTP time server as a stable clock reference. The Meinberg unit outputs a 1 PPS signal accurate to within $100 \mathrm{~ns}$ and is drift free.

\subsection{Experimental Results}

\subsubsection{Experiment 1: GPS Validation}

During December of 2005, we operated the SeaBED AUV offshore the coast of Woods Hole, MA using the R/V Tioga. For this set of experiments we deployed the AUV in approximately $15 \mathrm{~m}$ of water and programed the vehicle to execute a trajectory comprising two $100 \mathrm{~m}$ concentric boxes at a forward speed 


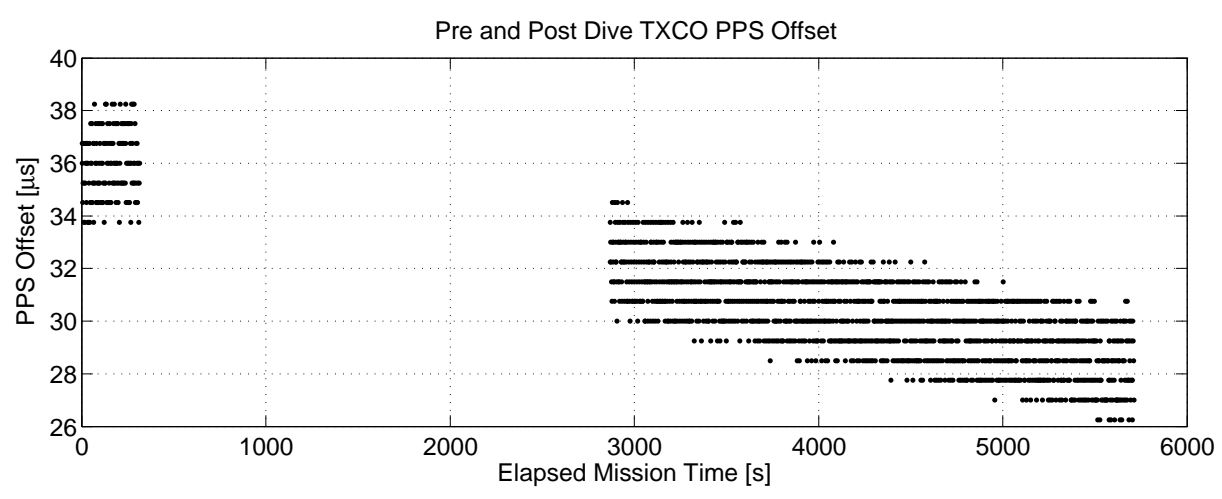

Figure 8: Pre and post-dive measured TXCO PPS offset with respect to GPS-derived absolute PPS timebase. The gap in the time series corresponds to when the AUV was subsea. During the course of the dive, the TXCO drifted by approximately $4 \mu$ s from an initial offset of $36 \mu$ s to $32 \mu \mathrm{s}$.

of $0.4 \mathrm{~m} / \mathrm{s}$. In this environment, the AUV had Doppler bottom-lock from the surface with a total mission time of approximately 1.5 hours; PPS clock-drift onboard the vehicle was less than $4 \mu$ s (Figure 8 ). The ship remained on anchor and OWTT ranges were broadcast at a periodic schedule of every 5 then 20 seconds. Figure 7 displays the raw OWTT data recorded between the AUV and ship; for comparison, independently measured two-way travel-time (Tracklink USBL) ranges are shown.

Equation (15) was used to calculate inter-ping DVL odometry covariance using settings of $3 \mathrm{~mm} / \mathrm{s}$ standard deviation for the body-frame surge/sway velocities (assumed isotropic) and $0.1^{\circ}$ standard deviation for the heading; these numbers were obtained from the respective manufacturer's manuals as being typical precisions. We set the OWTT ranges at $18.75 \mathrm{~cm}$ standard deviation, based upon the Micro-Modems $125 \mu \mathrm{s}$ TOA detection resolution. For ship GPS position error, we used the horizontal error estimated by the receiver and assumed it to be isotropic.

Figure 9 displays results from the MLE fusion of the OWTT pseudo-ranges and bottom-lock DVL odometry. The raw DVL trajectory, shown in blue, was obtained by forward Euler integration, using the GPS dropposition of the AUV as the origin. Shown in red is the globally referenced MLE derived trajectory; this result was post-processed offline. Shown in green is the independently measured post-dive GPS position of the AUV, which cross-validates the OWTT result. Figure 9(b) provides a plot of GPS referenced error versus time, showing good agreement between OWTT derived position and onboard GPS. 


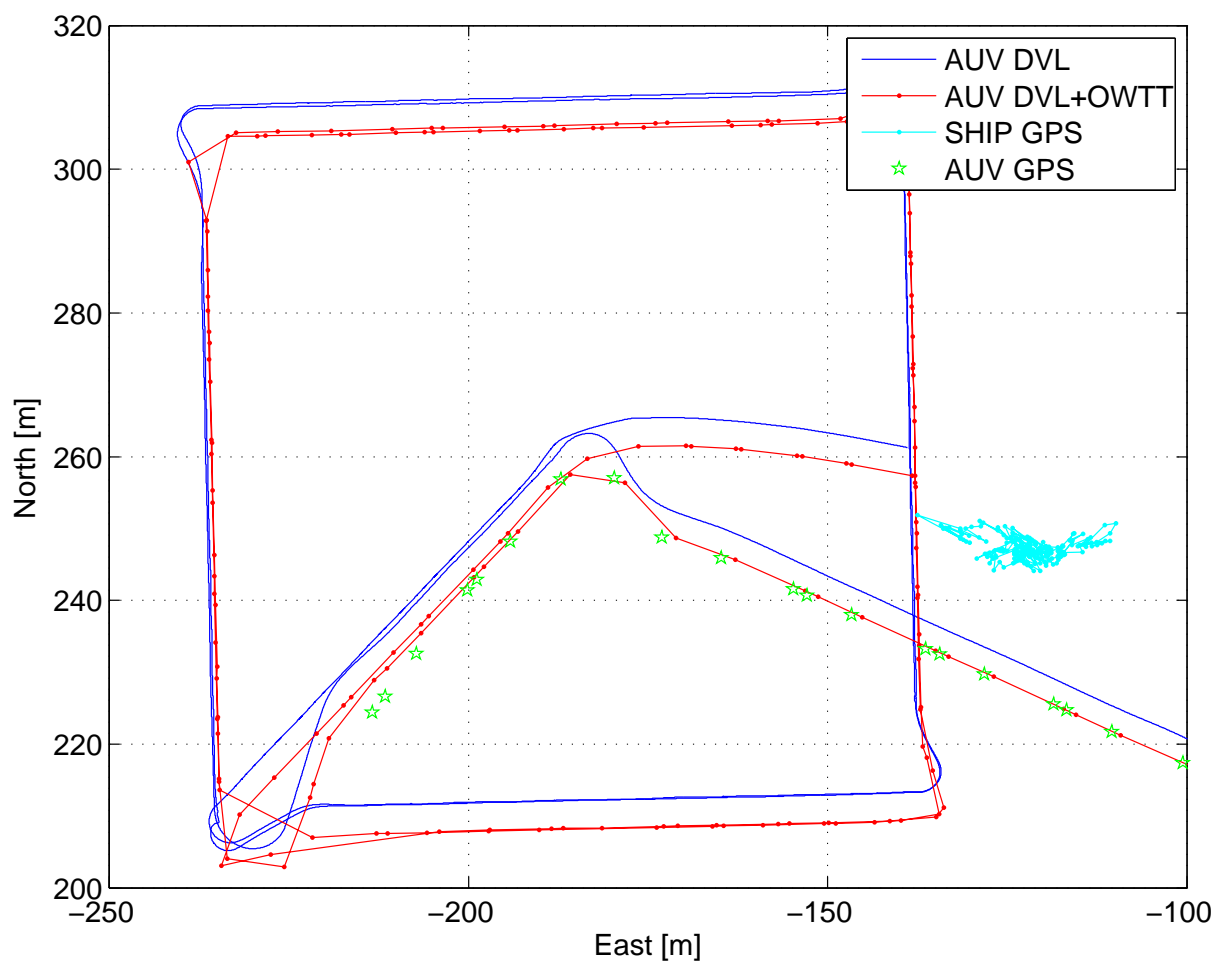

(a)

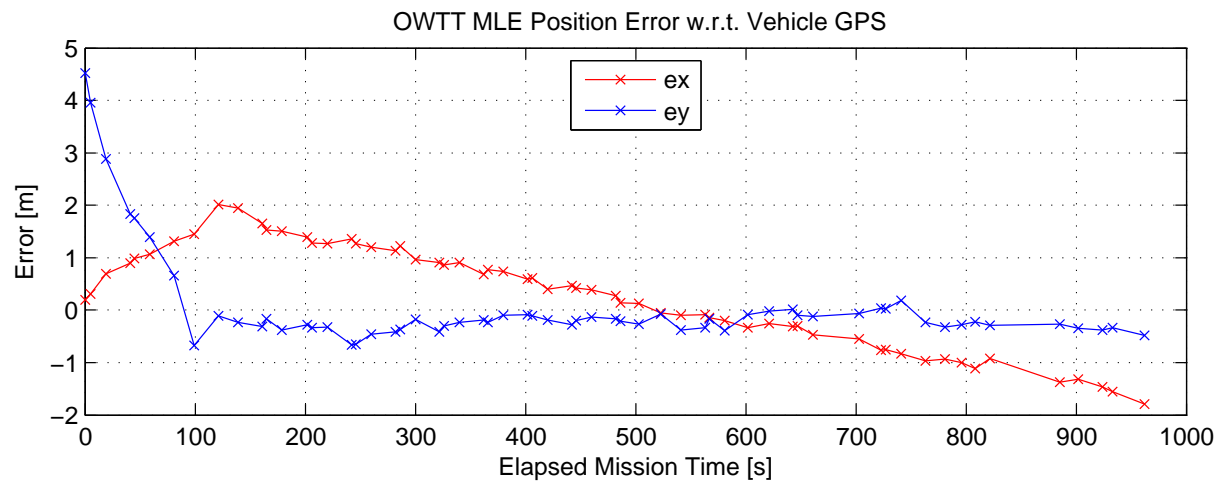

(b)

Figure 9: MLE sensor fusion results for a two node network consisting of a bottom-lock Doppler-aided AUV and a GPS-equipped surface ship. Each node is equipped with a WHOI Micro-Modem and onboard stable PPS source for synchronous clock communication/navigation. (a) The AUV swam two co-located box trajectories (100 m per side), each at a different depth set point while the ship remained anchored. Shown in blue is the raw DVL-derived AUV trajectory; in cyan is the GPS-derived ship position; in red is the globally referenced MLE AUV trajectory; and in green is the end-of-dive GPS-measured AUV position, which serves as an independent ground-truth. (b) Shown here is the error between end-of-dive OWTT-derived and GPSmeasured AUV position. 


\subsubsection{Experiment 2: LBL Validation}

In July of 2006, we operated aboard the Greek vessel R/V Aegeo as part of a joint WHOI / Hellenic Centre for Marine Research (HCMR) research expedition in the Mediterranean. This time we performed a longer series of OWTT experiments with the SeaBED AUV, deploying it for a mission just under 2 hours in duration. The water depth was approximately $50 \mathrm{~m}$ deep and the programed survey trajectory consisted of two grid-patterns: one oriented East-to-West and the other North-to-South. The survey bounding box was approximately $200 \mathrm{~m}$ on a side. We used the same measurement noise parameters as described in $\S 5.2 .1$. In addition, we deployed a two-transponder LBL net for independent position validation.

Figure 10 shows results comparing raw DVL, MLE-fused OWTT data, and cross-validated $12 \mathrm{kHz}$ LBL vehicle position. For this experiment the surface ship free-drifted about the survey site, occasionally motoring to get back on station; its trajectory is depicted by the cyan curve in Figure 10(a). Figure 10(b) shows a close up view of the re-navigated survey. The coarse agreement between OWTT MLE and LBL is visually evident, in contrast with the raw Doppler track whose error increases with time. Figures 10(c)/10(d) and 10(e)/10(f) show that the raw DVL trackline exhibits a time-dependent bias while the OWTT re-navigated trajectory does not exhibit a time-dependent bias.

\subsection{Discussion}

The experimental results from the two field tests corroborate that the DVL-aided OWTT navigation framework outperforms the DVL-alone navigation error - overall showing good agreement with the bounded-error, independent positioning methods provided by surface GPS and LBL. In particular, the time cumulative DVL drift bias has been removed via the fusion of the OWTT range constraints in the MLE framework. What is interesting to note is that not any single OWTT range measurement sufficiently constrains the vehicle's position, rather it places it on an annulus centered about the ship's broadcast position. It is through the vector displacement odometry provided by the DVL that three or more OWTT range measurements provide sufficient constraint to uniquely bound the XY position error of the vehicle. In the absence of bottom-lock DVL, the vector displacement odometry constraint could be derived from an INS or, with less fidelity, from a vehicle dynamic model; however, we have not yet independently corroborated the performance of either of those two alternative scenarios.

During both experimental tests, the communication protocol was symmetric in that the vehicle periodically transmitted OWTT packets up to the ship, while the ship frequently downlinked OWTT packets to the 


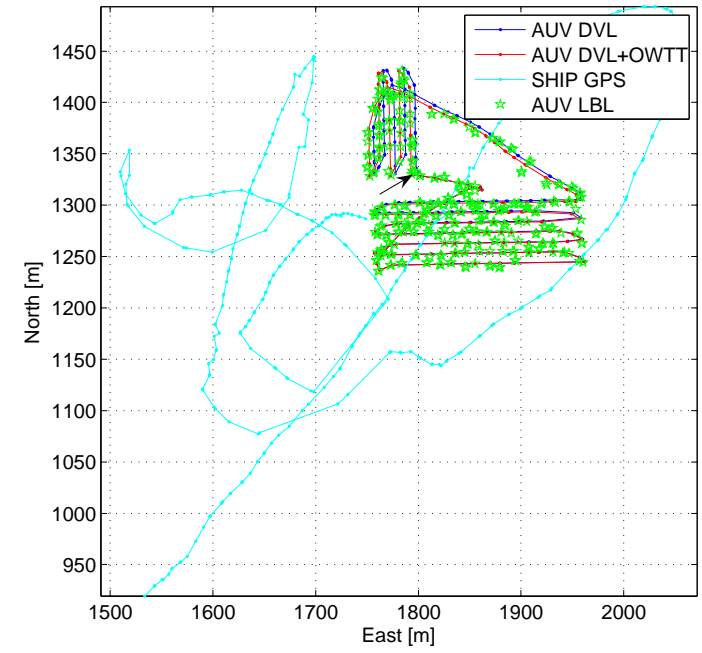

(a)

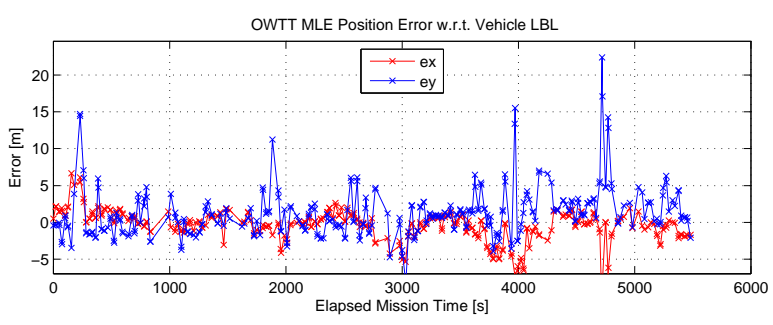

(c)
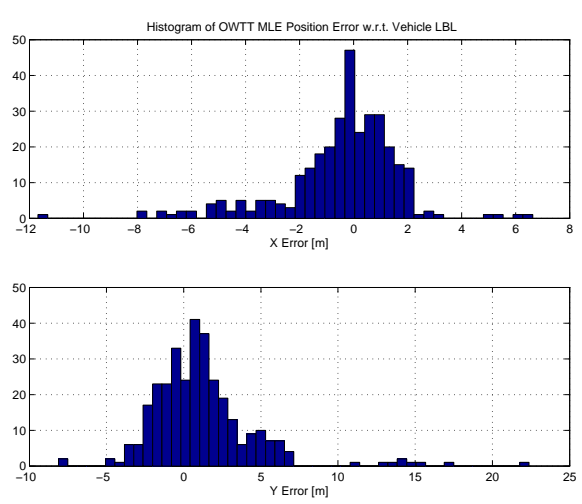

(e)

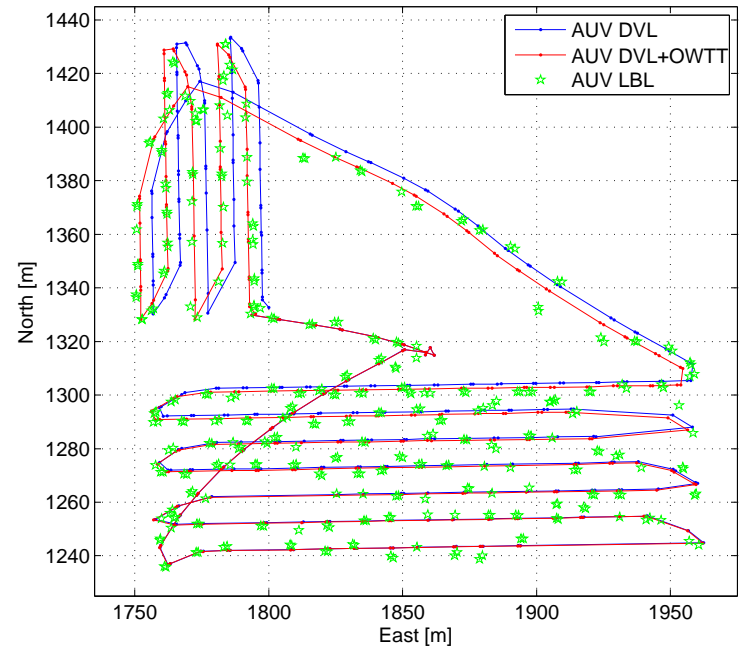

(b)

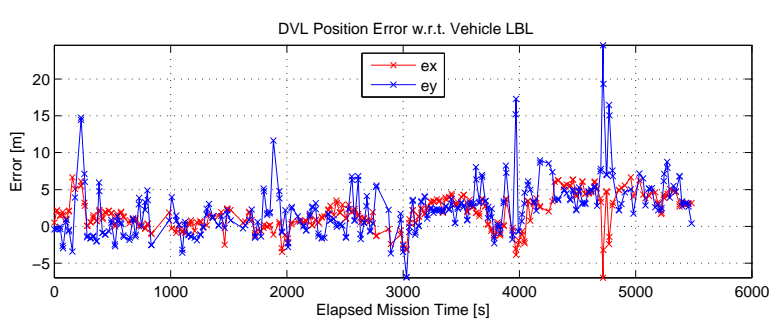

(d)
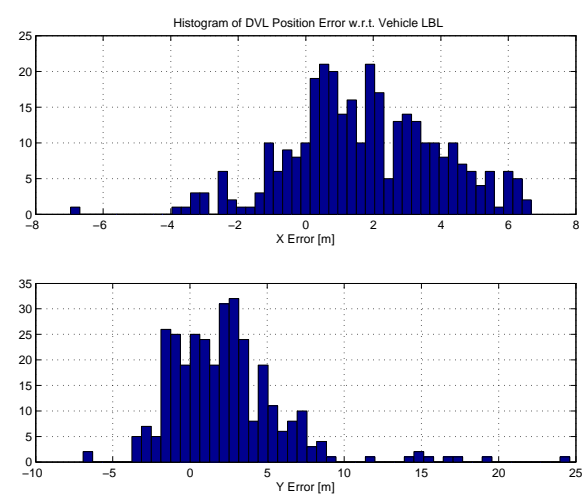

(f)

Figure 10: MLE sensor fusion results for a two-node configuration consisting of a GPS-equipped surface ship and bottom-lock Doppler-aided AUV. The survey took place in approximately $50 \mathrm{~m}$ of water depth. (a) The programed vehicle trajectory consisted of two grid-survey patterns: one oriented East-to-West and the other North-to-South. Shown in blue is the raw DVL-derived DR AUV trajectory; in cyan is the GPS-derived ship position; in red is the globally referenced MLE AUV trajectory; and in green is the $12 \mathrm{kHz}$ LBL-derived AUV position, which serves as an independent ground-truth. The mission starts and ends at approximately $(1794,1330)$, as indicated by the arrow. (b) A close up view of the vehicle trajectory. The discrepancy between raw DVL position and LBL increases with time. In contrast, the OWTT-aided trajectory agrees well with the independently measured LBL position. (c) and (d) These two plots show OWTT and raw DVL position error versus elapsed mission time; error is defined with respect to LBL. Again, note the zero-mean nature of OWTT-derived position versus the time dependent DVL error. X and Y error histograms are shown in (e) and (f). 
vehicle (both on a predefined schedule). In the proposed MLE estimation framework this makes no difference in the optimal post-processed re-navigation of the AUV since OWTT measurements are indexed between pairs of ship/vehicle states and hence can accommodate such an uplink/downlink scenario. For real-time subsea navigation of the AUV, a sliding time-window version of the batch MLE could be run on the vehicle using downlink OWTT measurements and navigational data to compute a running position fix. We are also currently pursuing a more computationally efficient recursive estimation formulation based upon an extended Kalman filter framework, and its dual - an extended information filter, as preliminarily reported in (Webster et al., 2009) and (Webster et al., 2010), respectively. Note that for efficient simultaneous multivehicle navigation, the ship should act as a master and primarily downlink to the passively listening vehicle fleet since under that scenario all passively receiving vehicles within acoustic range will measure the OWTT constraint independent of the number of vehicles in the water.

Another interesting point worth noting is that the relative ship/vehicle trajectory geometry plays an important role in the global positional observability obtained by OWTT constraints from a single moving reference beacon. Earlier work by Song (1999) for the range-only target tracking problem showed that the observer's trajectory must exhibit nonzero derivative dynamics of the target's trajectory, (i.e., if the target is traveling on a constant velocity trajectory, the observer's trajectory must exhibit nonzero acceleration). In practice, this means that the ship's trajectory should be nonzero and should change its heading course if a vehicle is conducting a linear trackline survey. Further study into optimal trajectory geometries for minimal global error is the subject of future work.

Also worth noting is that for dives exceeding one day in length, which are becoming typical of modern oceanographic AUVs, the modeling of clock drift will play a crucial role in effective use of the OWTT navigation framework. The experiments reported herein were of short duration, and hence clock drift could be ignored. On dives exceeding $14 \mathrm{hr}$ in length, we can expect clock-drift on the TXCO's employed in our system to approach $1 \mathrm{~ms}$ of time (worst case) and thereby introduce a range-bias of $1.5 \mathrm{~m}$ into the pseudo range constraint. This bias would be exhibited in all range measurements and strongly time-correlatedtherefore it may be possible to observe clock bias within the state estimation framework. Notionally, one simple solution would be to stop the ship and vehicle, exchange OWTT modem messages down and up, and by comparing the downlink OWTT to the uplink OWTT one could estimate the clock offset. In the case of either one or both vehicles moving this would become more difficult to measure as it would require knowledge of the range change due to inter-vehicle motion to be compensated for using odometry or other kinematic information. At this point in time, further study is required to investigate under what conditions 
clock-bias estimation may be feasible.

\section{Conclusions}

This article reported recent advances in the development of a synchronous-clock acoustic communication/navigation system for underwater vehicles. The long-term goal of this work is to enable the task of navigating a fleet of AUVs over geometrically unconstrained length scales with bounded-error commensurate with standard $12 \mathrm{kHz}$ LBL navigation. Toward that end, we have reported the development of a low power, stable clock system suitable for integration on AUVs. We have also formulated a principled maximumlikelihood fusion framework for combining OWTT pseudo-range measurements with vehicle-odometry for bounded-error navigation. For this purpose we presented a covariance analysis of bottom-lock Dopplerderived odometry. Results from two field experiments validating the OWTT maximum likelihood estimator framework were reported. Future research in this area will address the modeling of PPS clock drift, the use of water-lock DVL velocity odometry, and a decentralized estimation framework to support multi-vehicle navigation with inter-vehicle ranging.

\section{Acknowledgments}

This work was supported by the National Science Foundation under Award Numbers ATM-0427220, ATM0428122, and IIS-0746455, and in part by the National Oceanic and Atmospheric Administration Office of Ocean Exploration under Grant Number NA06OAR4600092.

\section{References}

Baccou, P. and Jouvencel, B. (2002). Homing and navigation using one transponder for AUV, post-processing comparisons results with long base-line navigation. In Proceedings of the IEEE International Conference on Robotics and Automation, volume 4, pages 4004-4009.

Bahr, A. and Leonard, J. (2006). Cooperative localization for autonomous underwater vehicles. In Proc. 10th Intl. Symp. on Experimental Robotics (ISER), pages 387-395, Rio de Janeiro, Brasil.

Bellingham, J., Deffenbaugh, M., Leonard, J., and Catipovic, J. (1994). Arctic under-ice survey operations. Unmanned Systems, 12:24-29.

Curcio, J., Leonard, J., Vaganay, J., Patrikalakis, A., Bahr, A., Battle, D., Schmidt, H., and Grund, M. 
(2005). Experiments in moving baseline navigation using autonomous surface craft. In Proceedings of the IEEE/MTS OCEANS Conference and Exhibition, pages 730-735, Washington, D.C.

Djugash, J. and Singh, S. (2008). A robust method of localization and mapping using only range. In Khatib, O., Kumar, V., and Pappas, G., editors, 11th International Symposium on Experimental Robotics, volume 54 of Springer Tracts for Advanced Robotics, pages 341-351. Springer.

Freitag, L., Grund, M., Partan, J., Ball, K., Singh, S., and Koski, P. (2005a). Multi-band acoustic modem for the communications and navigation aid AUV. In Proceedings of the IEEE/MTS OCEANS Conference and Exhibition, pages 1080-1085, Washington, D.C.

Freitag, L., Grund, M., Singh, S., Partan, J., Koski, P., and Ball, K. (2005b). The WHOI micro-modem: an acoustic communications and navigation system for multiple platforms. In Proceedings of the IEEE/MTS OCEANS Conference and Exhibition, pages 1086-1092, Washington, D.C.

Gadre, A. and Stilwell, D. (2004). Toward underwater navigation based on range measurements from a single location. In Proceedings of the IEEE International Conference on Robotics and Automation, volume 5, pages $4472-4477$.

Gadre, A. and Stilwell, D. (2005). A complete solution to underwater navigation in the presence of unknown currents based on range measurements from a single location. In Proceedings of the IEEE/RSJ International Conference on Intelligent Robots and Systems, pages 1420-1425.

Gaiffe, T. (2002). U-Phins: a FOG-based inertial navigation system developed specifically for AUV navigation and control. In Intl. Conf. on Underwater Intervention, New Orleans, LA.

Haralick, R. M. (1998). Propagating covariance in computer vision. Technical Report CITR-TR-29, Computer Science Department of The University of Auckland, CITR at Tamaki Campus, New Zealand.

Hunt, M., Marquet, W., Moller, D., Peal, K., Smith, W., and Spindel, R. (1974). An acoustic navigation system. Technical Report WHOI-74-6, Woods Hole Oceanographic Institution.

Kinsey, J. C., Eustice, R. M., and Whitcomb, L. L. (2006). Underwater vehicle navigation: recent advances and new challenges. In IFAC Conf. on Manoeuvring and Control of Marine Craft, Lisbon, Portugal.

Larsen, M. (2000a). High performance Doppler-inertial navigation-experimental results. In Proceedings of the IEEE/MTS OCEANS Conference and Exhibition, volume 2, pages 1449-1456, Providence, RI.

Larsen, M. (2000b). Synthetic long baseline navigation of underwater vehicles. In Proceedings of the IEEE/MTS OCEANS Conference and Exhibition, volume 3, pages 2043-2050.

Mills, D. (2006). Network time protocol version 4 reference and implementation guide. Technical Report 06-06-1, University of Delaware. 
Milne, P. (1983). Underwater acoustic positioning systems. Gulf Publishing Company, Houston.

Partan, J., Kurose, J., and Levine, B. N. (2007). A survey of practical issues in underwater networks. $A C M$ SIGMOBILE Mobile Computing and Communications Review, 11(4):23-33.

Poole, M. (2007). Wideband signals the future for acoustic positioning. Hydro International, 11(3):28-30.

Press, W., Teukolsky, S., Vetterling, W., and Flannery, B. (1992). Numerical recipes in C: the art of scientific computing. Cambridge University Press, 2nd edition.

RD Instruments (2001). Navigator ADCP/DVL users guide. San Diego, CA. P/N 957-6173-00.

Ristic, B., Arulampalam, S., and McCarthy, J. (2002). Target motion analysis using range-only measurements: algorithms, performance and application to ISAR data. Signal Processing, 82(2):273-296.

Ross, A. and Jouffroy, J. (2005). Remarks on the observability of single beacon underwater navigation. In Proceedings of the International Symposium on Unmanned Untethered Submersible Technology.

Rossby, T., Dorson, D., and Fontaine, J. (1986). The RAFOS system. Journal of Atmospheric and Oceanic Technology, 3:672-679.

Scherbatyuk, A. (1995). The AUV positioning using ranges from one transponder LBL. In Proceedings of the IEEE/MTS OCEANS Conference and Exhibition, volume 3, pages 1620-1623, San Diego, CA.

Singh, H., Bellingham, J., Hover, F., Lerner, S., Moran, B., von der Heydt, K., and Yoerger, D. (2001). Docking for an autonomous ocean sampling network. IEEE Journal of Oceanic Engineering, 26(4):498514.

Singh, H., Can, A., Eustice, R. M., Lerner, S., McPhee, N., Pizarro, O., and Roman, C. (2004). SeaBED AUV offers new platform for high-resolution imaging. EOS, Transactions of the American Geophysical Union, 85(31):289,294-295.

Singh, H., Catipovic, J., Eastwood, R., Freitag, L., Henriksen, H., Hover, F., Yoerger, D., Bellingham, J., and Moran, B. (1996). An integrated spproach to multiple AUV communications, navigation and docking. In Proceedings of the IEEE/MTS OCEANS Conference and Exhibition, volume 1, pages 59-64, Fort Lauderdale.

Singh, S., Grund, M., Bingham, B., Eustice, R. M., Singh, H., and Freitag, L. (2006). Underwater acoustic navigation with the WHOI micro-modem. In Proceedings of the IEEE/MTS OCEANS Conference and Exhibition, pages 1-4, Boston, MA.

Smith, S. and Kronen, D. (1997). Experimental results of an inexpensive short baseline acoustic positioning system for AUV navigation. In Proceedings of the IEEE/MTS OCEANS Conference and Exhibition, volume 1, pages 714-720, Halifax, Nova Scotia, Canada. 
Song, T. (1999). Observability of target tracking with range-only measurements. IEEE Journal of Oceanic Engineering, 24(24):383-387.

Vaganay, J., Baccou, P., and Jouvencel, B. (2000). Homing by acoustic ranging to a single beacon. In Proceedings of the IEEE/MTS OCEANS Conference and Exhibition, volume 2, pages 1457-1462.

Webster, S. E., Eustice, R. M., Singh, H., and Whitcomb, L. L. (2009). Preliminary deep water results in single-beacon one-way-travel-time acoustic navigation for underwater vehicles. In Proceedings of the IEEE/RSJ International Conference on Intelligent Robots and Systems, pages 2053-2060, St. Louis, MO.

Webster, S. E., Whitcomb, L. L., and Eustice, R. M. (2010). Preliminary results in decentralized estimation for single-beacon acoustic underwater navigation. In Proceedings of the Robotics: Science \& Systems Conference, Zaragoza, Spain.

Whitcomb, L., Yoerger, D., and Singh, H. (1998). Towards precision robotic maneuvering, survey and manipulation in unstructured undersea environments. In Proceedings of the International Symposium on Robotics Research, pages 45-54, Springer Verlag, London.

Zhou, X. S. and Roumeliotis, S. I. (2008). Robot-to-robot relative pose estimation from range measurements. IEEE Transactions on Robotics, 24(6):1379-1393. 